\title{
Improving mucosal anesthesia for awake endotracheal intubation with a novel method: a prospective, assessor-blinded, randomized controlled trial
}

Chunji Han ${ }^{1 \dagger}$, Peng $\mathrm{Li}^{2 \dagger}$, Zhenggang Guo ${ }^{3}$, Ying Guo ${ }^{1}$, Li Sun ${ }^{1}$, Gang Chen ${ }^{1}$, Xiaojue Qiu', Weidong Mi ${ }^{1}$, Changsheng Zhang ${ }^{1 *}$ id and Lorenzo Berra ${ }^{5}$

\begin{abstract}
Background: Topical anesthesia is a crucial step in awake endotracheal intubation for providing favorable intubation conditions. The standard of care technique for awake intubation at our institution, which consists of oropharyngeal tetracaine spray, can result in inadequate mucosal anesthesia. Therefore, we sought to compare the effectiveness of dyclonine hydrochloride mucilage to the standard of care tetracaine in achieving anesthesia of the upper airways for awake endotracheal intubation.

Methods: This is a randomized, assessor-blinded, prospective study. From Jun. 1st, 2019 to Aug. 1st, 2019, patients scheduled for either endoscopic submucosal dissection or peroral endoscopic myotomy were enrolled and randomly allocated into two groups after obtaining written informed consent: patients allocated to novel awake intubation care (Group N-AIC) received a single administration of oral dyclonine hydrochloride mucilage, whereas patients allocated to standard awake intubation care (Group S-AIC) received three oropharyngeal tetracaine sprays before transcricoid tetracaine injection before awake intubation. Mean arterial pressure (MAP), which was the primary outcome of this study, as well as heart rate (HR) were recorded throughout the procedure and compared between the two groups. Feeling of numbness, nausea, and intubation conditions after topical anesthesia were also assessed.

\footnotetext{
*Correspondence: powerzcs@126.com

${ }^{\dagger}$ Chunji Han and Peng Li contributed equally to this work.

${ }^{1}$ Anesthesia and Operation Center, The First Medical Center of Chinese PLA General Hospital, 28th Fuxing Rd., Haidian District, Beijing 100853, P. R. China

Full list of author information is available at the end of the article
}

(C) The Author(s). 2020 Open Access This article is licensed under a Creative Commons Attribution 4.0 International License, which permits use, sharing, adaptation, distribution and reproduction in any medium or format, as long as you give appropriate credit to the original author(s) and the source, provide a link to the Creative Commons licence, and indicate if changes were made. The images or other third party material in this article are included in the article's Creative Commons licence, unless indicated otherwise in a credit line to the material. If material is not included in the article's Creative Commons licence and your intended use is not permitted by statutory regulation or exceeds the permitted use, you will need to obtain permission directly from the copyright holder. To view a copy of this licence, visit http://creativecommons.org/licenses/by/4.0/ The Creative Commons Public Domain Dedication waiver (http://creativecommons.org/publicdomain/zero/1.0/) applies to the data made available in this article, unless otherwise stated in a credit line to the data. 


\begin{abstract}
(Continued from previous page)
Results: Sixty patients were enrolled and completed the study. Baseline MAP and HR were similar between the two groups. However, hemodynamic responses to intubation and gastrointestinal endoscopy, especially MAP, were significantly less elevated in Group N-AIC. The degree of numbness of the oropharyngeal mucosa after topical anesthesia did not differ between the two groups, neither did the feeling of nausea during laryngoscopy. The amount of pharyngeal secretions before intubation was less in Group N-AIC. Total intubation time was significantly shorter in Group N-AIC when compared to Group S-AIC (18.4 \pm 2.86 vs. $22.3 \pm 6.47, P<0.05)$. Extubation bucking was significantly less frequent in Group N-AIC (13.3\% vs. 76.7\%). Patients received in Group N-AIC had a lower rate of post-extubation sore throat compared to Group S-AIC (6.7\% vs. 43.3\%). No adverse side effects attributable to either tetracaine or dyclonine were observed in this study.
\end{abstract}

Conclusions: In awake endotracheal intubation, novel care using oral dyclonine hydrochloride mucilage can provide more favorable mucosal anesthesia and better intubation conditions compared to standard of care practice using oropharyngeal tetracaine spray.

Trial registration: ChiCTR1900023151. Date of registration: May 14th, 2019.

Keywords: Topical anesthesia, Awake endotracheal intubation, Dyclonine, tetracaine

\section{Key points}

Question: Does novel awake intubation care using oral dyclonine hydrochloride mucilage improve mucosal anesthesia for awake endotracheal intubation?

Findings: Novel awake intubation care using $10 \mathrm{ml}$ of oral dyclonine hydrochloride mucilage is associated with fewer pharyngeal secretions, shorter intubation time, minor mean arterial pressure fluctuations, lower extubation bucking and sore throat rate perioperatively than the standard awake intubation care using three oropharyngeal tetracaine sprays (1\%).

Meaning: In awake endotracheal intubation, novel care using oral dyclonine hydrochloride mucilage can provide more favorable intubation conditions and more stable hemodynamics than the standard of care using oropharyngeal tetracaine spray.

\section{Background}

Awake intubation consists of placing an endotracheal tube in the trachea while a patient continues to breathe spontaneously. This technique can be utilized in many different situations to help control a potentially unstable airway. However, awake intubation can be a difficult, timeconsuming maneuver for the anesthesiologist and an unpleasant experience for the patient [1]. Satisfactory execution of awake intubation needs both moderate sedation and sufficient topical anesthesia. Thus, topical anesthesia of the upper airways, including the oropharyngeal and subglottic tracheal mucosa, is a crucial element in providing adequate comfort for the patient throughout the procedure [2].

Traditionally, the standard of care at our institution for topical anesthesia of the oropharyngeal mucosa is with oropharyngeal tetracaine $(0.5 \sim 1 \%)$ or lidocaine (4\%) sprays, while topical anesthesia of the subglottic tracheal mucosa is provided by tetracaine $(2 \%)$ transcricoid or intratracheal injection [3-6]. Tetracaine is a potent local anesthetic commonly used as the standard of care for awake intubation in many health care institutions. Application of tetracaine can effectively blunt the cough reflex and provide topical anesthesia for procedures requiring mucosal anesthesia, such as bronchoscopy and endotracheal intubation. However, the standard of care for awake intubation is potentially complicated by the undesirable properties of tetracaine sprays, including its narrow safety profile, sialogogic effects, and bitter taste [7].

Dyclonine hydrochloride, a relatively new and different chemical compound with local anesthetic properties, was initially adopted for endoscopic procedures to reduce pain, nausea, patient movements, and to lubricate the gastroscope [8]. Multiple reports have shown that dyclonine is more effective than tetracaine and lidocaine in providing adequate mucosal anesthesia $[9,10]$. In daily clinical practice, the oral application of dyclonine hydrochloride mucilage does not involve as many complicated steps as tetracaine sprays do in the current standard of care regimen for awake intubation. Additionally, dyclonine can be safely used in patients with documented allergy to bupivacaine and procaine [11]. However, dyclonine is rarely utilized as a topical anesthetic for patients requiring awake endotracheal intubation. Therefore, it is worth further investigating whether dyclonine could be a potential mucosal anesthetic for use in awake intubation.

In this prospective, assessor-blinded, randomized controlled trial, the authors compared the mucosal anesthetic efficacy of oral dyclonine hydrochloride mucilage to the current standard of care, pharyngeal tetracaine sprays, for performing awake endotracheal intubation.

\section{Methods}

This was a prospective, randomized controlled trial performed at the endoscopy center, First Medical Center of 
Chinese PLA General Hospital. This study was approved by the Ethical Committee of Chinese PLA General Hospital (\#2019-088-01), and written informed consent was obtained from all subjects participating in the trial. The trial was registered prior to patient enrollment at the Chinese Clinical Trial Registry (ChiCTR1900023151, Principal investigator: Changsheng Zhang, Date of registration: May 14th, 2019). This manuscript adheres to the applicable CONSORT guidelines.

\section{Study population}

From Jun. 1st, 2019 to Aug. 1st, 2019, 60 patients aged 20-65 years were enrolled in this study. Inclusion criteria included patients meeting criteria for American Society of Anesthesiology (ASA) Class I or II who were scheduled for endoscopic submucosal dissection (ESD) or peroral endoscopic myotomy (POEM) under general anesthesia. Patients were excluded if they had one or more of the following criteria: a history of asthma, known allergy to study drugs, anticipated difficult intubation, history of documented chronic organ failure, hypertension, ischemic heart disease, atrioventricular block, incomplete or partial heart blocks, application of vasoactive drugs perioperatively.

\section{Study procedures}

Patients were randomly allocated to the standard awake intubation care group (Group S-AIC) or novel awake intubation care group (Group N-AIC) in an assessorblinded fashion based on a computer-generated code. The anesthesiologists participating in this study had at least 5 years of experience as attending physicians at our institution. In this study, topical anesthesia administration to the upper airway was performed alone by an anesthesia nurse to ensure that the anesthesiologist, the clinical investigator, and the data analyst were all blinded to the study grouping.

After arriving in the operation room, venous access was obtained with an 18-gauge cannula placed in the left forearm. Electrocardiogram, pulse oximetry, and noninvasive blood pressure (cuff placed on the right upper arm) were continuously monitored. The standard of care for awake intubation at our institution is to perform topical anesthesia using tetracaine sprays of both the oropharyngeal mucosa and the tracheal mucosa after Bispectral index (BIS) has reached $80-85$. The patient is then intubated with a video laryngoscope, video stylet, and flexible fiberoptic scope. Thus, in Group S-AIC, moderate sedation of the patient was provided with intravenous midazolam $(0.03 \mathrm{mg} / \mathrm{kg})$ and fentanyl $(2 \mu \mathrm{g} /$ $\mathrm{kg}$ ) boluses. After adequate sedation was achieved, patients received oropharyngeal tetracaine (Chengdu Tiantaishan Pharmaceutical Co., Ltd., China) spray three times every $2 \mathrm{~min}(9$ sprays and 2 intervals of $2 \mathrm{~min}$ in total. See supplemental video). First, the soft palate was sprayed. Then, the radix linguae were sprayed while the patient was instructed to pronounce ha. Finally, the epiglottis was sprayed with the guidance of delicate video laryngoscopy. The total volume of tetracaine (1\%) used for oropharyngeal spray was $0.5 \mathrm{ml}$. In Group N-AIC, patients received oral administration of dyclonine hydrochloride mucilage $(10 \mathrm{mg} / 10 \mathrm{ml}$, Yangtze River Pharmaceutical Co. Ltd., China) for topical anesthesia of the oropharyngeal mucosa using the same sedation index. The first $2 \mathrm{ml}$ were slowly swallowed as a test-dose to rule out possible allergic reaction. After $2 \mathrm{~min}$, the remaining $8 \mathrm{ml}$ were administered and kept in the oropharynx for $3 \mathrm{~min}$ before swallowing.

The degree of oropharynx mucosal numbness was evaluated $2 \mathrm{~min}$ after both study procedures. After obtaining adequate pharyngeal anesthesia, needle cricothyroidotomy was performed in both groups, and $2 \mathrm{ml}$ of tetracaine $(2 \%)$ were injected to provide topical anesthesia of the subglottic tracheal mucosa. Three minutes later, all patients were instructed to swallow all the secretions and drug residues in the mouth and were intubated with a video laryngoscope while spontaneously breathing. The total time and number of attempted intubations were recorded.

After tube insertion, a cuff pressure between 24 and $28 \mathrm{~cm} \mathrm{H}_{2} \mathrm{O}$ was maintained using an aneroid manometer to provide an adequate seal of the airway. Patients were instructed to place themselves in the left lateral position with the tube in place. General anesthesia induction was achieved by initiating target-controlled infusion (TCI) of propofol (Marsh model, target effect-site concentration of $2-4 \mu \mathrm{g} / \mathrm{kg} \cdot \mathrm{min}$ ) and remifentanil (Minto model, target effect-site concentration of 3-4 ng $/ \mathrm{kg} \cdot \mathrm{min})$. The propofol and remifentanil targets were adjusted to maintain target BIS values between 40 and 60 during the entire procedure. All patients were mechanically ventilated to maintain end-tidal $\mathrm{CO}_{2}\left(\mathrm{EtCO}_{2}\right)$ between 32 and 36 $\mathrm{mmHg}$ during the surgery.

Patients' hemodynamic parameters, mean arterial pressure (MAP) and heart rate (HR), were recorded at the following time points: $10 \mathrm{~min}$ after arriving in the endoscope room (T0), before the needle cricothyroidotomy (T1), immediately after intubation (T2), $5 \mathrm{~min}$ after intubation (T3), $3 \mathrm{~min}$ after left lateral positioning (T4), and immediately after extubation (T5). The degree of oropharyngeal mucosal numbness was defined as invalid, slight numbness, numbness, or significant numbness. The severity of nausea during laryngoscopy was assessed using a verbal numerical rating scale of $0-10 \quad(0=$ no feeling of nausea, $10=$ severe nausea). The best view obtained by video laryngoscope in each subject was described as that which visualized the glottis or the epiglottis. The amount of secretions before intubation 
was classified as few, medium or heavy secretions, and the amount of suctioning required before intubation was recorded. The patient's tolerance of endotracheal tube presence was assessed by the anesthesiologist during the patient self-positioning into left lateral decubitus as good, medium, or bad. Bucking response and presence of sore throat were recorded at extubation. The severity of sore throat at $24 \mathrm{~h}$ and $48 \mathrm{~h}$ after surgery was assessed using a verbal numerical rating scale of $0-10(0=$ no sore throat, $10=$ worst sore throat imaginable).

\section{Statistical analysis}

We anticipated enrolling 30 subjects $(27+10 \%$ possible dropouts) in both groups. According to our pilot study, sample size calculations showed that 27 patients were needed in both groups in order to detect a difference in MAP immediately after intubation between the two groups of $7.7 \mathrm{mmHg}$ (standard deviation $9.8 \mathrm{mmHg}$ ) with a power of 0.8 and a two-sided $p$ value of less than 0.05 .

The statistical analysis was conducted using Statistical Package for Social Sciences (SPSS Inc., Chicago, IL, Version 17.0 for Windows). Results are expressed as means and standard deviations, medians and ranges, or numbers and percentages. The comparison of normally distributed continuous variables between the groups was performed using $t$-test. For time-dependent changes, repeated measures analysis of variance was applied. Normality of data was checked by measures of skewness and Kolmogorov Smirnov tests of normality. Nominal categorical data between the groups were compared using the chi-squared test or Fisher's exact test as appropriate.
Ordinal categorical variables and non-normal distribution of continuous variables were compared using the Mann-Whitney $U$-test. A $p$ value of less than 0.05 was considered statistically significant.

\section{Results}

A total of 60 patients who underwent ESD or POEM were enrolled in this study. No patients were excluded from further analysis (Fig. 1). No adverse side effects of either method for awake intubation care was observed in this study. The demographic data did not differ between the two groups with regard to age and body mass index (BMI). The distribution as per sex, ASA status, and surgery type was similar in both groups, and the mean duration of surgery was comparable in both groups and statistically non-significant (Table 1).

Perioperative mean arterial pressure is more stable in patients who underwent novel awake intubation care MAP and HR measurements of the six perioperative time points are summarized in Figs. 2 and 3. The baseline (T0 and T1) MAP and HR were comparable between the two groups. However, there was an overall statistically significant differences among the two groups regarding MAP immediately after intubation (T2) and subsequent time points T3 and T4 $(P<0.0083$, Bonferroni corrected significance level). The novel awake intubation care using dyclonine was found to significantly reduce MAP at intubation and left lateral positioning when compared to standard awake intubation care. However, although the mean HR in the N-AIC group was slightly lower at T2, T3, T4, and T5 as compared

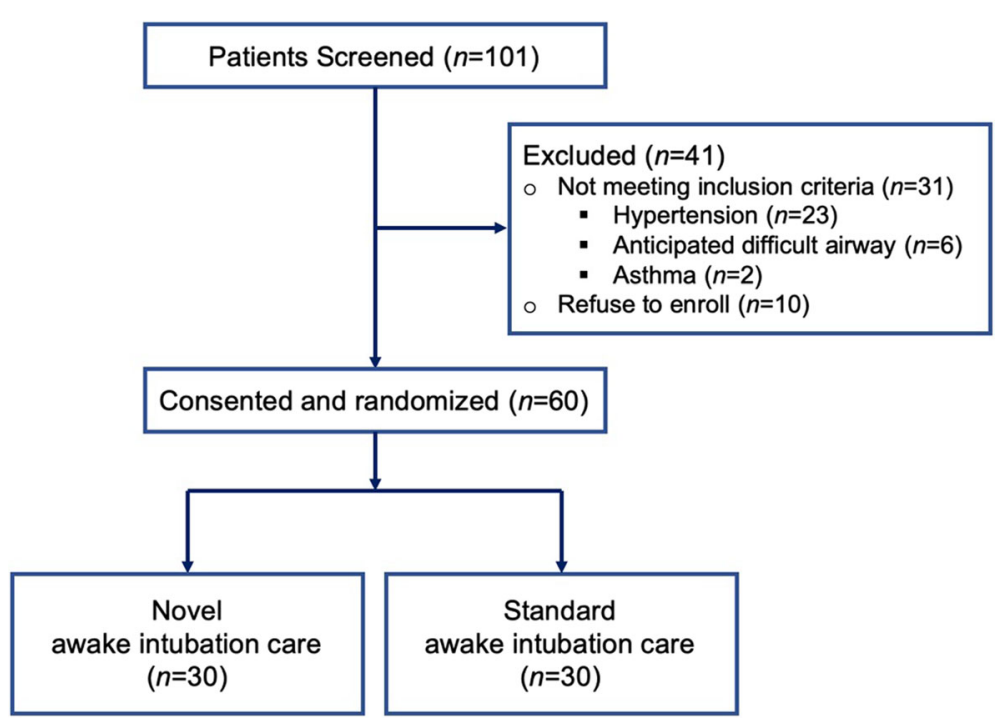

Fig. 1 Flow chart of 101 consecutive patients scheduled for endoscopic submucosal dissection (ESD) or peroral endoscopic myotomy (POEM) under general anesthesia during the study period. After 41 patients were excluded for reasons stated above, 30 patients were randomized to novel awake intubation care, and 30 patients were randomized to standard awake intubation care 
Table 1 Patient characteristics

\begin{tabular}{llll}
\hline Characteristic & Group N-AIC & Group S-AIC & P value \\
\hline Age, Median (Quartile) & $55(48-61)$ & $54.5(44-60)$ & $23.78(3.54)$ \\
BMI, mean (SD) & $24.02(2.94)$ & $17(56.67)$ \\
Sex, Male (\%) & $16(53.3)$ & $6 / 24$ \\
ASA (I/II) & $6 / 24$ & \\
Surgical procedure & & $25(83.33)$ \\
$\quad$ ESD (\%) & $25(83.33)$ & $5(16.67)$ \\
POEM (\%) & $5(16.67)$ & $62(45-106)$ \\
Duration of surgery (min), Median (Quartile) & $63.5(40-86)$ & $500(400-500)$ \\
Fluids infused (ml), Median (Quartile) & $500(446-500)$ &
\end{tabular}

$B M I$ Body mass index, ASA American Society of Anesthesiology, ESD Endoscopic submucosal dissection, POEM Peroral endoscopic myotomy, N-AIC Novel awake intubation care, S-AIC Standard awake intubation care

a Two-sample $t$ test. Chi-squared test

${ }^{b}$ chi-squared test

with the S-AIC group, the difference was not statistically significant $(P=0.124)$ (Table 2).

Patients who underwent novel awake intubation care had fewer secretions in the oropharynx and shorter intubation duration

The amount of secretions in patients' oropharynx before intubation was less in the N-AIC group compared to the $\mathrm{S}$-AIC group $(P=0.01)$. Favorable intubation conditions (visualized glottis) were reported in 30 patients in the $\mathrm{N}$ AIC group and 27 patients in the $\mathrm{S}$-AIC group $(P=$ $0.237)$. No patients in the N-AIC group required suctioning before intubation, and only three patients in the $\mathrm{S}$-AIC group required upper airway suctioning before intubation $(P=0.237)$. The total time of intubation was significantly shorter in the N-AIC group $(18.4 \pm 2.86$ vs. $22.3 \pm 6.47, P<0.05$, Fig. 4). All the patients in the $\mathrm{N}$ -

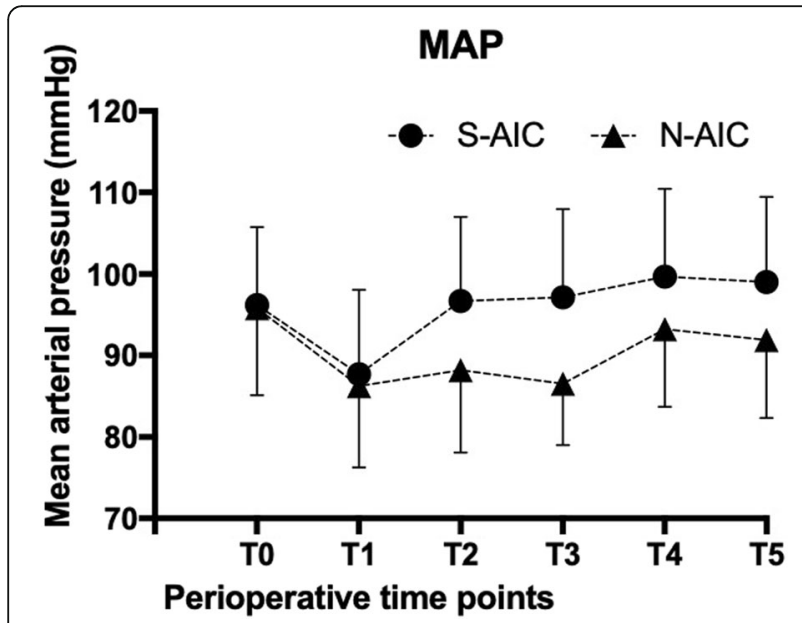

Fig. 2 Results of mean arterial pressure (MAP) of the six perioperative time points. Error bars are $+/-$ standard error of the mean. There were significant differences between groups at the time points of T2, T3, T4, T5 $(P<0.05)$
AIC group were successfully intubated at the first attempt, while only one patient in the S-AIC group required two attempts. The endotracheal tube was well tolerated in lateral decubitus in most patients, except for two patients in the S-AIC group whose tolerance was reported as "medium".

\section{Novel awake intubation care did not improve patients'} subjective sensation but reduced extubation bucking and sore throat at $24 \mathrm{~h}$ after extubation

The degree of numbness of the oropharyngeal mucosa after topical anesthesia and the feeling of nausea during laryngoscopy were not different between the two groups ( $P=0.546$ and $P=0.317$, respectively). However, only $13.3 \%$ of patients who received N-AIC had bucking at extubation, compared to $76.7 \%$ with S-AIC $(P<0.001)$. Patients in the N-AIC had a much lower rate of

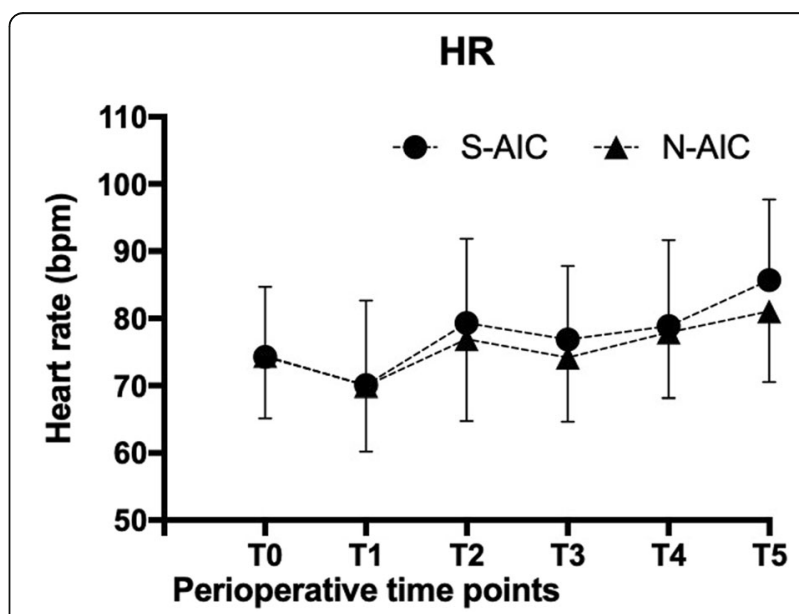

Fig. 3 Results of heart rate (HR) of the six perioperative time points. Error bars are +/- standard error of the mean. No significant differences were found between groups 
Table 2 Comparison of MAP and HR between the study groups at various time points

\begin{tabular}{|c|c|c|c|c|c|c|}
\hline \multirow{2}{*}{$\begin{array}{l}\text { Time } \\
\text { points }\end{array}$} & \multirow[t]{2}{*}{ Parameter } & \multicolumn{2}{|c|}{ Group N-AIC } & \multicolumn{2}{|c|}{ Group S-AIC } & \multirow[t]{2}{*}{$P$ Value $^{\mathrm{a}}$} \\
\hline & & Mean & SD & Mean & SD & \\
\hline \multirow[t]{2}{*}{ T0 } & MAP $(\mathrm{mmHg})$ & 95.83 & 10.74 & 96.17 & 9.57 & 0.899 \\
\hline & HR (bpm) & 74.43 & 9.25 & 74.27 & 10.46 & 0.948 \\
\hline \multirow[t]{2}{*}{ T1 } & MAP $(\mathrm{mmHg})$ & 86.23 & 9.98 & 87.70 & 10.39 & 0.579 \\
\hline & HR (bpm) & 69.97 & 9.78 & 70.07 & 12.62 & 0.630 \\
\hline \multirow[t]{2}{*}{$\mathrm{T} 2$} & MAP $(\mathrm{mmHg})$ & 88.17 & 10.11 & 96.67 & 10.32 & $0.002^{*}$ \\
\hline & HR (bpm) & 76.90 & 12.16 & 79.33 & 12.49 & 0.448 \\
\hline \multirow[t]{2}{*}{ T3 } & MAP $(\mathrm{mmHg})$ & 86.53 & 7.49 & 97.13 & 10.79 & $0.000^{*}$ \\
\hline & HR (bpm) & 74.17 & 9.51 & 76.87 & 10.97 & 0.312 \\
\hline \multirow[t]{2}{*}{ T4 } & MAP $(\mathrm{mmHg})$ & 93.23 & 9.50 & 99.70 & 10.75 & $0.000^{*}$ \\
\hline & HR (bpm) & 77.90 & 9.77 & 78.87 & 12.757 & 0.743 \\
\hline \multirow[t]{2}{*}{ T5 } & MAP $(\mathrm{mmHg})$ & 91.90 & 9.54 & 99.03 & 10.42 & $0.008^{*}$ \\
\hline & HR (bpm) & 81.10 & 10.58 & 85.73 & 11.942 & 0.120 \\
\hline
\end{tabular}

MAP Mean arterial pressure, $H R$ Heart rate, SD Standard deviation * $P<0.05$ considered statistically significant

${ }^{a}$ Repeated measurement analysis of variance

extubation sore throat than the S-AIC group (6.7\% vs. $43.3 \%, P<0.001)$. At $24 \mathrm{~h}$ after surgery, the severity of sore throat was significantly lower in the N-AIC group than the S-AIC group $(0[0-1]$ vs. $3[0-4], P=0.001)$. However, this difference in throat soreness between the two groups did not achieve statistical significance at $48 \mathrm{~h}$ after surgery $(0[0-0]$ vs. $0[0-0], P=0.31)$.

\section{Discussion}

In this prospective, randomized, controlled trial, we compared the effect of two different types of topical anesthesia for awake intubation care, using oral dyclonine hydrochloride mucilage $(10 \mathrm{mg} / 10 \mathrm{ml})$ or tetracaine spray $(1 \%, 0.5 \mathrm{ml})$. We found that awake intubation care

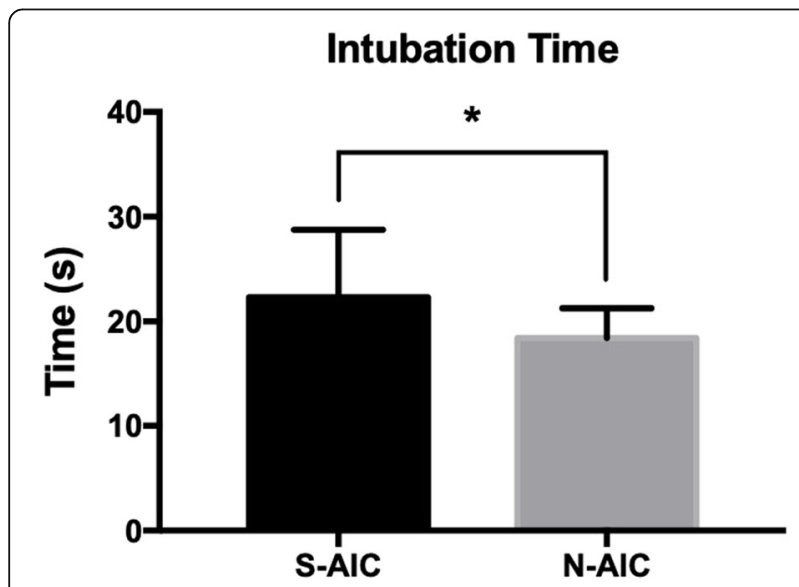

Fig. 4 Intubation time between groups. Each vertical bar represents the mean \pm standard error ( $n=30$ in each group). The total time of intubation was significantly shorter in the N-AIC group $\left({ }^{*} P<0.05\right)$ using oral dyclonine hydrochloride mucilage provides more favorable intubation conditions and better hemodynamics during the perioperative periods than standard awake intubation care.

The reason why awake intubation is a routine method for patients who undergo general anesthesia at our endoscopy center is because it is a relatively safer method for airway management, which could decrease potential aspiration risks and free from muscle relaxants perioperatively. Thus, satisfactory methods for awake intubation are always taken into account. Topical anesthesia of the oropharyngeal mucosa is of crucial importance before awake intubation, and can provide the patient with a relatively comfortable feeling during the laryngoscopic examination. Adequate topical anesthesia of the oropharyngeal mucosa could also result in better cooperation from the patient. Induction of amnesic sedation followed by awake intubation is a common technique at our institution. Given the rapid absorption of tetracaine in the pharynx, tetracaine spray is routinely used to provide topical anesthesia for fiberoptic tracheal intubation and other procedures requiring mucosal anesthesia. In addition, tetracaine frequently provides topical anesthesia for gastrointestinal and ocular procedures. It is reported that the maximum effective concentration of tetracaine is $1 \%$, with a latent period of 1.1 min and a duration of $55.5 \mathrm{~min}$ [12].

However, in our experience, topical tetracaine is far from being the ideal mucosal anesthetic due to the following two reasons. First, the bitter taste and feeling of nausea after oropharyngeal application cause patients to regurgitate or swallow the drug, which significantly reduces the amount of drug acting on the mucosa. Additionally, anesthesiologists who are not experienced in administering topical anesthesia of the oropharyngeal mucosa often leave some parts of the mucosa unanesthetized, and thus still sensitive to stimulation. Stevens et al. tested the effects of tetracaine inhalation before intubation. He found that a small dose of nebulized tetracaine may completely coat mucosal surfaces and significantly attenuates the hemodynamic response to tracheal intubation [13]. Therefore, complete coating of mucosal surfaces is a crucial factor of successful topical anesthesia. This may explain why the effects of tetracaine spray used in the S-AIC group is not as good as dyclonine hydrochloride mucilage used in N-AIC in attenuating the hemodynamic response of intubation.

Dyclonine hydrochloride mucilage is a topical anesthetic that reversibly binds to activated sodium channels on the neuronal membrane, thereby decreasing the neuronal membrane's permeability to sodium ions, leading to an increased threshold for excitation [14]. It very effectively produces topical anesthesia and lubricates the mucosal surfaces for gastrointestinal endoscopy 
and endotracheal intubation $[15,16]$. When applied to mucus membranes, the onset of topical anesthesia is 2$10 \mathrm{~min}$ and lasts for 20-30 min [12]. Beginning in 1983, the safety and effectiveness of dyclonine were recognized as superior to lidocaine and tetracaine for awake intubation [17]. In 1997, Bacon et al. reported the use of oral and nebulized dyclonine for topical anesthesia of the airway to facilitate awake intubation in a patient with a stated allergy to bupivacaine and procaine [11]. In our study, we found that dyclonine hydrochloride mucilage may have a more prolonged effect than reported because the incidence of extubation bucking and sore throat was significantly lower in the N-AIC group, factors that contributed in providing a better anesthesia and intubation experience to patients.

In this study, we did not find any difference in the subjective sensation of topical anesthesia between the two kinds of awake intubation care, which demonstrate the similar anesthetic effects of both drugs after proper administration. However, patients who received N-AIC had a significantly better hemodynamic profile during and after the procedure, suggesting that dyclonine mucilage significantly attenuates hemodynamic distress induced by laryngoscopy and endotracheal intubation, especially if compared to tetracaine.

Notably, the duration of the intubation process was significantly shorter in patients who received novel awake intubation care. The defoaming effect of dyclonine hydrochloride mucilage, which eliminate the mucous bubbles in the oral-pharyngeal cavity, leads to a better view and fewer visible secretions during the videolaryngoscopic examination [18]. Thus, dyclonine mucilage administration not only provides better mucosal anesthesia (i.e., better attenuates hemodynamic response of laryngoscopy and intubation, is an efficacious anesthetic effect, and leads to excellent patient cooperation), but can also provide better intubation conditions (i.e., fewer visually obstructive secretions and faster intubation).

However, the limitations of our study must be acknowledged. The subjective assessments of the intubation condition by anesthesiologists, and the subjective sensation of the numbness of oropharynx mucosa, nausea and sore throat reported by the patients may lead to subjective bias, which may be the underlying reason for lack of statistically significant differences in some of these parameters. The sedation level prior to intubation was not recorded, which may lead to a difference in subjective sensation during and after topical anesthesia among the patients. Future studies are expected to reveal the effects of dyclonine as a nasal mucosal topical anesthetic during nasotracheal intubation and could provide more comprehensive information for its clinical application.

\section{Conclusion}

In awake endotracheal intubation, novel care using oral dyclonine hydrochloride mucilage can provide more favorable mucosal anesthesia and better intubation conditions than standard of care using oropharyngeal tetracaine sprays.

\section{Supplementary Information}

The online version contains supplementary material available at https://doi. org/10.1186/s12871-020-01210-8.

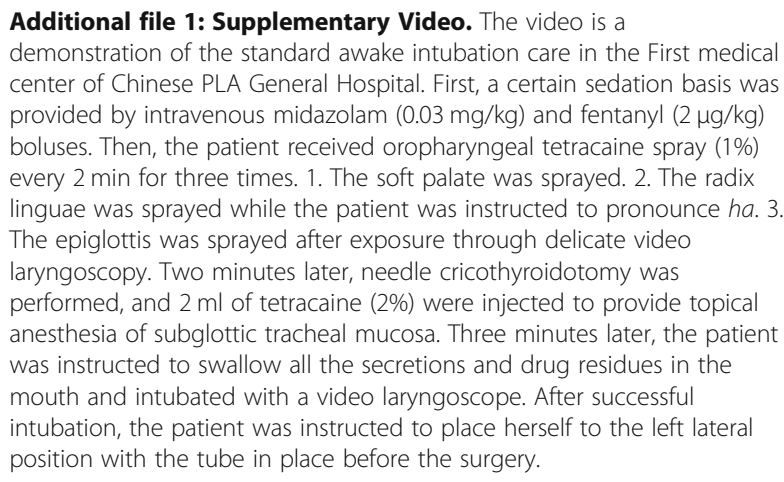
center of Chinese PLA General Hospital. First, a certain sedation basis was provided by intravenous midazolam $(0.03 \mathrm{mg} / \mathrm{kg})$ and fentanyl $(2 \mu \mathrm{g} / \mathrm{kg})$ boluses. Then, the patient received oropharyngeal tetracaine spray (1\%) every 2 min for three times. 1. The soft palate was sprayed. 2 . The radix linguae was sprayed while the patient was instructed to pronounce ha. 3 . The epiglottis was sprayed after exposure through delicate video laryngoscopy. Two minutes later, needle cricothyroidotomy was performed, and $2 \mathrm{ml}$ of tetracaine (2\%) were injected to provide topical anesthesia of subglottic tracheal mucosa. Three minutes later, the patient was instructed to swallow all the secretions and drug residues in the mouth and intubated with a video laryngoscope. After successful intubation, the patient was instructed to place herself to the left lateral position with the tube in place before the surgery.

\begin{abstract}
Abbreviations
S-AIC: Standard awake intubation care; N-AIC: Novel awake intubation care: ASA: American Society of Anesthesiology; ESD: Endoscopic submucosal dissection; POEM: Peroral endoscopic myotomy; BIS: Bispectral index; TCl: Target-controlled infusion; $\mathrm{EtCO}_{2}$ : End-tidal $\mathrm{CO}_{2}$; MAP: Mean arterial pressure; HR: Heart rate
\end{abstract}

\section{Acknowledgements}

The authors thank Dr. Massimiliano Pirrone from the Department of Anesthesia and Critical Care, Fondazione IRCCS Ca' Granda Ospedale Maggiore Policlinico, University of Milan, Italy, and Dr. David AE Imber from the Department of Pediatrics, Boston Children's Hospital, United States, for professional writing services and preparing the manuscript.

\section{Authors' contributions}

All authors have read and approved the manuscript. The detailed contribution of each author were listed below. $\mathrm{CH}$ : This author helped design the study, collect and analyze data, interpret data, and prepare the manuscript. PL: This author helped design the study, collect and analyze data, interpret data, and prepare the manuscript. ZG: This author helped search the literature, analyze the data, interpret data, and prepare the manuscript. YG: This author helped search the literature, analyze the data, interpret data, and prepare the manuscript. LS: This author helped design the study, collect and analyze data, and interpret data. GC: This author helped collect and analyze data, and interpret data. XQ: This author helped collect and analyze data. WM: This author helped design the study, collect and analyze data, and interpret data. CZ: This author helped search the literature, design the study, collect and analyze data, interpret data, prepare the manuscript, and collect funds. LB: This author helped search the literature, design the study, interpret data, and prepare the manuscript.

\section{Funding}

This work was supported by the National Natural Science Foundation of China [Grant No.81901096], which provide financial support for the design and conduct of the research, interpretation of data, and preparation of the article.

Availability of data and materials

The datasets used and/or analyzed during the current study are available from the corresponding author on reasonable request. 


\section{Ethics approval and consent to participate}

This study was approved by the Ethical Committee of Chinese PLA General Hospital (\#2019-088-01), and written informed consent was obtained from all subjects participating in the trial. The trial was registered prior to patient enrollment at the Chinese Clinical Trial Registry (ChiCTR1900023151, Principal investigator: Changsheng Zhang, Date of registration: May 14th, 2019).

\section{Consent for publication}

Written consent to publish the attached video was obtained from study participants, and the pdf of the consent was submitted as an additional file.

\section{Competing interests}

The authors declare that they have no competing interests.

\section{Author details}

'Anesthesia and Operation Center, The First Medical Center of Chinese PLA General Hospital, 28th Fuxing Rd., Haidian District, Beijing 100853, P. R. China. ${ }^{2}$ Department of Anesthesia, The Sixth Medical Center of Chinese PLA General Hospital, Beijing, China. ${ }^{3}$ Department of Anesthesiology, Peking University Shougang Hospital, Beijing 100144, China. ${ }^{4}$ Department of Gastroenterology, The First Medical Center of Chinese PLA General Hospital, Beijing, China. ${ }^{5}$ Department of Anesthesia, Critical Care and Pain Medicine, Massachusetts General Hospital, Boston, MA, USA.

Received: 7 July 2020 Accepted: 22 November 2020

Published online: 14 December 2020

\section{References}

1. Xue FS, Liu QJ. Tracheal intubation awake or under anesthesia for potential difficult airway: look before you leap. Chin Med J. 2018;131(6):753-6.

2. Simmons ST, Schleich AR. Airway regional anesthesia for awake fiberoptic intubation. Reg Anesth Pain Med. 2002;27(2):180-92.

3. Madineh $\mathrm{H}$, Amani S, Kabiri M, Karimi B. Evaluation of the anesthetic effect of nasal mucosa with tetracaine $0.5 \%$ on hemodynamic changes and postoperative pain of septoplasty: a randomized controlled trial. J Adv Pharm Technol Res. 2017:8(4):116-9.

4. Xue FS, Liu HP, He N, Xu YC, Yang QY, Liao X, Xu XZ, Guo XL, Zhang YM. Spray-as-you-go airway topical anesthesia in patients with a difficult airway: a randomized, assessor blinded comparison of 2 and $4 \%$ lidocaine. Anesth Analg. 2009;108(2):536-43.

5. Ji M, Tao J, Cheng M, Wang Q. Endotracheal administration of sufentanil and tetracaine during awake fiberoptic Intubation. Am J Ther. 2016;23(1): e92-7.

6. Peng Liang WY, Liu B. Application of tetracaine spray through thyrocricoid puncture before intubation in intensive care unit. Chin J Respir Crit Care Med. 2012;11(1):76-9.

7. Morris MJ, Kwon HP, Zanders TB. Monitoring, sedation, and anesthesia for flexible fiberoptic bronchoscopy. In: Haranath SP, editor. Global perspectives on bronchoscopy. Rijeka: InTech; 2012.

8. Ping RS, White JG, Spear LB. Dyclonine hydrochloride as a topical anesthetic in dentistry; a preliminary report. Oral Surg Oral Med Oral Pathol. 1957;10(6): 623-6.

9. Jichao S, Cuida M, Mingxing C, Yunyun W, Dongdong Z. Oral dyclonine hydrochloride mucilage versus tetracaine spray in electronic flexible laryngoscopy: a prospective, randomized controlled trial. Am J Otolaryngol. 2016;37(2):169-71.

10. Groeben H, Grosswendt T, Silvanus MT, Pavlakovic G, Peters J. Airway anesthesia alone does not explain attenuation of histamine-induced bronchospasm by local anesthetics: a comparison of lidocaine, ropivacaine, and dyclonine. Anesthesiology. 2001;94(3):423-8 discussion 425A-426A.

11. Bacon GS, Lyons TR, Wood SH. Dyclonine hydrochloride for airway anesthesia: awake endotracheal intubation in a patient with suspected local anesthetic allergy. Anesthesiology. 1997;86(5):1206-7.

12. Adriani J, Zepernick R. Clinical effectiveness of drugs used for topical anesthesia. JAMA. 1964;188:711-6.

13. Stevens JB, Vories PA, Walker SC. Nebulized tetracaine attenuates the hemodynamic response to tracheal intubation. Acta Anaesthesiol Scand. 1996:40(6):757-9.

14. Dyclonine Hydrochloride (Code C66875). In. NClthesaurus. https://ncit.nci. nih.gov/ncitbrowser/ConceptReport.jsp?dictionary=NCl_Thesaurus\&ns= ncit\&code $=$ C66875.
15. Levine DS, Blount PL, Rudolph RE, Reid BJ. Safety of a systematic endoscopic biopsy protocol in patients with Barrett's esophagus. Am J Gastroenterol. 2000;95(5):1152-7.

16. Groeben H, Schlicht M, Stieglitz S, Pavlakovic G, Peters J. Both local anesthetics and salbutamol pretreatment affect reflex bronchoconstriction in volunteers with asthma undergoing awake fiberoptic intubation. Anesthesiology. 2002;97(6):1445-50.

17. Adriani J, Savoie A, Naraghi M. Scope and limitations of topical anesthetics in anesthesiology prictis. Anesthesiol Rev. 1983;7:10-5.

18. Sun T, Gu X, Lu H, Chen M. Oral preparation of dyclonine hydrochloride. In: United States. Taizhou: Yangtze River Pharmaceutical (Group) Co., Ltd.; 2010.

\section{Publisher's Note}

Springer Nature remains neutral with regard to jurisdictional claims in published maps and institutional affiliations.
Ready to submit your research? Choose BMC and benefit from:

- fast, convenient online submission

- thorough peer review by experienced researchers in your field

- rapid publication on acceptance

- support for research data, including large and complex data types

- gold Open Access which fosters wider collaboration and increased citations

- maximum visibility for your research: over $100 \mathrm{M}$ website views per year

At $\mathrm{BMC}$, research is always in progress.

Learn more biomedcentral.com/submissions 